\title{
L'Etat face aux communautés
}

\section{Christophe Jaffrelot}

\section{OpenEdition}

\section{Journals}

\section{Édition électronique}

URL : http://journals.openedition.org/conflits/511

DOI : $10.4000 /$ conflits. 511

ISSN : $1777-5345$

Éditeur :

CCLS - Centre d'études sur les conflits lilberté et sécurité, L'Harmattan

\section{Édition imprimée}

Date de publication : 15 octobre 1994

ISSN : 1157-996X

Référence électronique

Christophe Jaffrelot, "L'Etat face aux communautés », Cultures \& Conflits [En ligne], 15-16 | automnehiver 1994, mis en ligne le 15 mars 2006, consulté le 30 mars 2021. URL : http://

journals.openedition.org/conflits/511; DOI : https://doi.org/10.4000/conflits.511

Ce document a été généré automatiquement le 30 mars 2021.

Creative Commons License 


\title{
L'Etat face aux communautés
}

\author{
Christophe Jaffrelot
}

1 Le "communautarisme", traduction française de "communalism", plus courant dans le vocabulaire des sciences sociales anglo-saxonnes, désigne des groupes culturels partageant la même langue, la même religion et/ou les mêmes traits raciaux. Mais, définir ainsi les communautés à partir de caractéristiques culturelles ne revient pas à faire une lecture culturaliste du phénomène, telle que celle proposée par le courant "primordialiste" (pour lequel les identités collectives procèdent directement de " donnés " culturels ${ }^{1}$ ). Les communautés ne constituent pas ici des entités transhistoriques qui entreraient toute armée en politique. Le "communautarisme" est donc bien comme 1'indique le suffixe attaché au mot - une idéologie, c'est à dire une construction moderne et largement stratégique qui émerge nécessairement en relation avec la formation ou les transformations de l'Etat. Chacun des articles réunis dans ce numéro illustre à sa façon cette problématique même lorsqu'il adopte une perspective historique et remonte très loin dans le temps pour analyser justement le caractère construit du communautarisme.

2 Cependant dire que le communautarisme est un phénomène idéologique moderne ne suffit pas à le définir; c'est même à ce stade que surgit la question la plus difficile. Comment, en effet, distinguer cet " isme " des autres du même " genre ", comme le nationalisme et le particularisme pour n'en citer que deux? La question est d'autant plus délicate que les termes utilisés sont eux-mêmes des enjeux politiques. Des "nationalistes" auto-proclamés stigmatisent ainsi des "communalistes" dont l'action mine à leurs yeux l'unité du pays et bien des communautés cherchent par contre à revendiquer le statut de nations de manière à légitimer leurs aspirations à l'indépendance.

3 Communautarisme, nationalisme et particularisme La distinction entre " nationalisme " et " communautarisme " (ou communalisme) repose chez certains auteurs sur un rapport contradictoire aux valeurs individualistes. Pour Louis Dumont, " le communalisme diffère du nationalisme par la place que semble y prendre la religion " 2 considérée ici comme indissociable d'un univers holiste. Cette thèse s'inscrit dans une perspective ouverte par Marcel Mauss selon lequel la nation ne peut se développer que 
dans un contexte individualiste ${ }^{3}$. Cette conception est discutable dans les termes mêmes du modèle de L. Dumont qui oppose volontiers un nationalisme universaliste et individualiste, dont l'archétype est fourni par la France, et un "nationalisme ethnique", qui correspond davantage au cas allemand, où l'accent est mis sur les liens culturels et parfois de sang ${ }^{4}$. Dès lors comment distinguer le "communautarisme" du nationalisme ethnique?

Un élément de réponse réside sans doute dans les modalités d'insertion de la communauté dans un ensemble social et politique plus vaste. Le terme "communautarisme" s'applique en effet de façon privilégiée à des groupes culturels qui ne représentent qu'une composante parmi d'autres, d'un Etat multi-culturel et qui même, parfois, vivent en inter-action de sorte qu'ils se perçoivent dans certains cas comme des sous-ensembles de la nation et non comme des nations. Cette intégration relative ne signifie pas que la communauté ne puisse pas évoluer vers une forme de nationalisme ethnique et formuler pour finir des revendications indépendantistes - il s'agit au contraire d'un cas de figure fréquent qu'illustrent plusieurs articles de ce numéro - mais dans une acception idéal-typique du " communautarisme ", le groupe est inscrit dans un cadre national dont les références visent à transcender les clivages culturels au nom d'une identité englobante: les nationalismes libanais, mauricien, indien, yougoslave ... ont ou ont eu leur raison d'être, par rapport à des ennemis extérieurs (notamment au moment des luttes anti-coloniales) et les communautés sont dans cette configuration, censées fournir leur apport particulier à ces constructions qui sont davantage que la somme de ces composantes et qui affichent souvent une vocation universalistes. La maxime indienne répétée à l'envi dans de nombreux Etats pluriethniques " d'unité dans la diversité " renvoie à ce schéma.

La différence entre le " communautarisme" et le "particularisme " découle en partie de cette caractérisation des communautés comme composantes plus ou moins bien intégrées d'ensembles plus vastes. Le particularisme consiste en effet souvent à revendiquer la reconnaissance d'une spécificité par 1'Etat tandis que le communautarisme implique que cette étape est déjà réalisée et que les revendications portent sur davantage de concessions encore. Dans certains cas les communautés sont même institutionnalisées à travers le système fédéral (cas de l'ex-Yougoslavie) ou les techniques de représentation électorales (Liban, Maurice). Cet essai de typologie, qui est aussi un effort de clarification terminologique dans un domaine où le sens des concepts est souvent trop idiosyncrasique ou polémique, montre combien les différences peuvent être ténues entre des notions voisines. Cet exercice confirme en tout cas que l'on ne peut s'intéresser à ces questions sans rencontrer aussitôt l'Etat.

6 Les (contre)stratégies " ethniques " de l'Etat Le communautarisme se caractérise d'abord par sa prétention à opposer à la relation citoyenne une autre allégeance qui se veut prioritaire. Il remet, par là, en cause la construction d'un espace public et le principe même d'une société politique transcendant les spécificités culturelles. Si la constitution des communautés en forces sociales et politiques peut ainsi apparaître comme une menace adressée à l'Etat, ce dernier peut toutefois aussi chercher à les utiliser en vue de conforter ou de rétablir son pouvoir. Ce point de vue est sans doute complémentaire du précédent dans différents cas de figure. Le plus courant, semble-til, est celui où l'Etat s'efforce de diviser les communautés pour mieux régner; il peut alors utiliser une mobilisation identitaire de type extrémiste pour déstabiliser un rival politique représentant la même communauté mais sur un registre modéré. Le cas de 
l'Etat indien dans ses relations avec les sikhs du Pendjab illustre bien cette configuration. Ce genre de manipulation se traduit cependant par une nouvelle " communalisation " de l'Etat. Ce changement peut n'être que de degré dans les Etats où ces communautés sont reconnues de façon plus ou moins institutionnelle; il peut aussi être de nature là où l'Etat affiche (ou affichait) une vocation universaliste et laïque. Dans tous les cas, l'usage (contre)stratégique du communautarisme est contraire à la notion d'un Etat transcendant les clivages de la société.

7 La dimension internationale L'analyse des rapports entre Etats et communautés demande à être resituée dans le contexte international. Cette approche concerne l'impact de " ceux de l'extérieur " du point de vue transnational et le rôle des Etats voisins (ou plus lointains) d'un point de vue internationaliste plus classique. S'agissant du premier volet deux propositions peuvent être avancées et demandent à être testées. D'une part les communautés apparaissent comme des menaces d'autant plus grandes pour l'Etat, qu'elles bénéficient de soutiens à l'étranger. D'autre part, " ceux de l'extérieur " peuvent, à l'inverse, soutenir l'Etat dans ses efforts de survie, y compris au plan financier. Du point de vue international, il paraît important de prendre en compte l'attitude des Etats voisins abritant ou non des ressortissants de la même communauté que celle qui se mobilise dans un pays donné. Par ailleurs les Etats voisins peuvent alimenter des mouvements séparatistes, qu'ils comportent ou non des éléments de la communauté mobilisée. Enfin l'efficacité d'un mouvement communautaire et la démonstration de sa puissance supposent souvent une énonciation internationale de son identité et de ses buts et donc une internationalisation de son action de contestation.

\section{NOTES}

1. Cette interprétation "primordialiste" d'abord développée par C. Geertz (" The integrative revolution - Primordial sentiments and civil politics in the new states ", in C. Geertz (ed.), Old societies and new states - The quest for modernity in Asia and Africa, New York, The Free Press of Glencoe, 1963, pp. 105-157) a acquis une branche extrême que les pères fondateurs désapprouveraient sans aucun doute à travers l'approche sociobiologique représentée notamment par P. Van Den Berghe (voir notamment de cet auteur "Race and ethnicity : a sociobiological perspective", Ethnic and racial studies, vol. 1, oct. 1978.)

2. L. Dumont, "Nationalisme et communalisme", appendice D à Homo Hierarchicus, Paris, Gallimard, 1966, p. 378.

3. M. Mauss, "La nation", in Oeuvres, tome 3, Paris, Minuit, 1969, pp. 573-625.

4. L. Dumont, Essais sur l'individualisme, Paris, Le Seuil, 1983, pp. 115-131. 
INDEX

Mots-clés : Etat-nation, sciences politiques, identité 